
en el conocimiento sociotécnico: Marco teórico para estudiar conflictos medioambientales. Revista de Sociología, 34(1), 4-20. doi: 10.5354/0719-

\title{
Asimetría en el conocimiento sociotécnico: Marco teórico para estudiar conflictos medioambientales
}

\author{
[Asymmetry in socio-technical knowledge: A theoretical framework for the study of \\ environmental conflicts]
}

\author{
Cristián Parker (iD \& José Miguel Pérez Valdivia (DD \\ Universidad de Santiago de Chile
}

\begin{abstract}
Resumen
El presente artículo tiene como objetivo proponer un marco teórico y metodológico para el análisis del factor conocimiento sociotécnico en los procesos de evaluación ambiental, teniendo como referente lo que sucede generalmente en la evaluación de los megaproyectos de infraestructura, energía o minería. En particular, nuestro propósito es profundizar en un enfoque teórico sobre la construcción social del conocimiento socioambiental y sociotécnico, sobre las asimetrías de los actores sociales involucrados y el riesgo de la tecnocracia que inhibe la participación. Luego proponemos un marco metodológico para la investigación de las expresiones discursivas y semióticas de los discursos que contienen los conocimientos negociados en procesos de evaluación ambiental. Se propone este marco que aproxime a una adecuada comprensión y manejo de las asimetrías en el conocimiento sociotécnico, como elemento que ha sido poco estudiado y que, sin embargo, tiene relevancia en el inicio, desarrollo y desenlace de los conflictos socioambientales.
\end{abstract}

Palabras clave: conflictos socioambientales, conocimiento sociotécnico, evaluación de impacto ambiental, participación ciudadana, análisis semiótico.

\begin{abstract}
The objective of the present article is to propose a theoretical and methodological framework for the analysis of socio-technical knowledge as a factor in environmental assessment processes. This is done in the context of the evaluation procedures typically employed as part of infrastructure, energy and mining megaprojects. In particular, we will explore a theoretical approach that addresses the social construction of socio-environmental and sociotechnical knowledge, as well as the asymmetries of the social actors involved and the risk of the formation of a technocracy that inhibits participation. We propose a methodological framework for the study and analysis of the discursive and semiotic expressions of the discourses within which negotiated knowledge may be found during environmental assessment processes. The framework is proposed as a means of identifying and managing asymmetries in socio-technical knowledge. It is an issue which has been addressed by few studies to date, despite its relevance to the emergence, development and outcome of socio-environmental conflicts.
\end{abstract}

Keywords: socio-environmental conflicts, sociotechnical knowledge, environmental impact assessment, citizen participation, semiotic analysis.

Contacto: La comunicación sobre este artículo debe ser enviada a Cristián Parker, email: cristian.parker@usach.cl Financiamiento: Fondo Nacional de Desarrollo Científico y Tecnológico (FONDECYT Regular N 1181065). 


\section{INTRODUCCIÓN}

En los conflictos socioambientales intervienen un conjunto de factores entre los cuales cabe mencionar a aquellos vinculados a los procesos de participación en las evaluaciones ambientales y la intervención del factor conocimiento en dichas evaluaciones y conflictos. Ambas dimensiones pueden ser distinguidas analíticamente, pero están estrechamente relacionadas, como intentaremos mostrar en este trabajo. Se ha estudiado mucho acerca de la participación ciudadana en los procesos de evaluación ambiental (Delamaza, Maillet, \& Martínez Neira, 2017), o acerca de su ausencia o deficiencias, que en muchas ocasiones está marcando los conflictos.

Sin embargo, son escasos los estudios que examinan la forma en cómo el factor conocimiento -especialmente el sociotécnicointerviene en los procesos evaluativos y como muchas veces influye directamente en la generación del conflicto o en su resolución. Nuestra propuesta parte del supuesto de que la asimetría en el conocimiento sociotécnico entre los actores, en el escenario y en los procesos de evaluación ambiental, resulta ser un factor decisivo en cuanto a la gobernanza ambiental y la resolución democrática de los conflictos ambientales en torno a los megaproyectos.

Participación ciudadana en evaluación ambiental y la emergencia de conflictos
Desde comienzos de la década de los 90, la transición hacia la democracia luego de 17 años de dictadura militar trajo consigo una serie de políticas de apertura a la participación ciudadana, dentro de las cuales se consideró la política medioambiental del país. El elemento más sustancial fue la formulación de la ley 19,300 sobre bases generales del medio ambiente, la que incluía la integración de la participación ciudadana en las actividades relativas a la protección del medio ambiente, la cual se irá ampliando a medida que se tienen una serie de sucesivas reformas (Moraga Sariego, 2017).

Desde mediados del 2000 comienza una proliferación de conflictos socioambientales en América Latina, contando en Chile, solo entre 2005 y 2014, más de 100 casos (Delamaza et al., 2017). Desde la promulgación de la ley 19,300 hasta la actualidad, se han desarrollado 135 conflictos socioambientales, de los cuales 30 permanecen activos (Instituto Nacional de Derechos Humanos [INDH], 2019). Esta acumulación de conflictos derivó en una crisis de legitimidad de las instituciones vinculadas a la protección del medio ambiente (Costa Cordella, 2012).

Hacia el año 2010, la principal institución encargada de velar por el medio ambiente, la CONAMA (Comisión Nacional para el Medio Ambiente), pasó a constituirse como un ministerio autónomo, ampliando a su capacidad de acción, creando el Servicio de Evaluación 
Ambiental (encargado de aplicar el sistema de evaluación ambiental) y los Tribunales Ambientales (Barandiaran, 2016; Cordero \& Vargas, 2016), entregando herramientas para la participación ciudadana, pero la toma de decisiones seguía estando en mano de las autoridades de turno, sin generar un mecanismo de participación vinculante efectiva, primando la utilización de los criterios técnicos para la autorización de los proyectos (Cordero \& Vargas, 2016; Cuadra Montoya, 2012).

El estudio de los conflictos destaca que existen diversos actores socioambientales en presencia, cuya relación está marcada por la asimetría. Generalmente las empresas y las instituciones gubernamentales cuentan con mayor poder que las comunidades locales afectadas por los proyectos. La ventaja de las empresas por sobre las comunidades a la hora de la aprobación de los proyectos también se enmarca en la legislación ambiental chilena que está fuertemente marcada por la construcción de leyes dentro del marco del modelo neoliberal (Tecklin, Bauer, \& Prieto, 2011), fenómeno que se puede observar en diversos conflictos medioambientales a nivel latinoamericano (Acselrad, das Neves Bezerra, \& Muñoz Gaviria, 2010).

El factor conocimiento y los conflictos socioambientales

Los problemas ambientales no son únicamente factores externos que impactan a los seres humanos, como si estos no tuvieran ninguna injerencia en ellos. Para la sociología ambiental los humanos son parte del ambiente, por tanto el ambiente y la sociedad deben comprenderse plenamente en una relación mutua (King \& McCarthy, 2014). Hay muchos problemas que los humanos han provocado y que también han tratado de resolver, pero muchas incomprensiones acerca de la relación hombre/naturaleza han llevado a una serie de fenómenos como el cambio climático, los innumerables casos de contaminación, pérdida de biodiversidad, agotamiento de recursos y especies y, en general, desastres ambientales (Jorgenson, Dunlap, \& Clark, 2014), que están afectando al planeta de manera inédita y nuestro país no es una excepción. Frente a estos problemas los países desarrollados, desde los años 60 (Coria, 2008; Glasson, Therivel, \& Chadwick, 2005), han implementado formas y mecanismos de evaluación ambiental de tal manera de evaluar ex ante el probable impacto ambiental de los proyectos y programas públicos y privados, aprobarlos, generar recomendaciones para su mitigación cuando corresponde y/o rectificaciones, o sencillamente rechazar proyectos (García Leyton, 2004).

La experiencia histórica reciente en Chile, y en numerosos otros casos latinoamericanos (Costa Cordella, 2012; Lostarnau et al., 2011; Silva, 2012), nos lleva a pensar que los procesos de evaluación ambiental se enfrentan con ciertas dificultades que no sólo son de carácter técnico o político. Se ha estudiado cómo los relatos expertos de la realidad física han entrado en conflicto con el conocimiento local. Se ha mostrado que, en lugar de que el conocimiento local sea rutinariamente inferior o defectuoso, este resulta ser más sensible a las realidades 
locales (Latta, 2007; Yearley, 2000). De esta manera ha quedado en evidencia que coexisten diversas formas de conocimientos que se ponen en juego en los procesos de evaluación ambiental y generalmente esos conocimientos no están distribuidos equitativamente, o bien, se trata de tipos de conocimientos (expertos / no expertos; universales / locales; científicos / de sentido común, etc.) ubicados en esferas de jerarquía distintas, ocupando escalones inferiores aquellos conocimientos que se encuentran producidos y reproducidos en las comunidades.

Elementos de un marco teórico: La construcción social del conocimiento ambiental

Desde el punto de vista sociológico, el problema del conocimiento no reside solamente en cuanto a la variación empírica de este en las sociedades humanas. El problema central es cómo cualquier cuerpo de conocimiento llega a ser establecido como realidad. Por tanto, no interesa la validez o no del conocimiento, sino los procesos por los cuales una determinada realidad queda establecida y cristalizada para los actores sociales (Berger \& Luckmann, 1966|1999). La realidad de la vida cotidiana se presenta en forma ordenada, intersubjetiva, como algo dado que se estructura en el espacio y el tiempo. La realidad humana, y por ende el conocimiento, es posible por el proceso de objetivación, y el más importante de ellos se da en el mundo de signos y lenguajes. El lenguaje trasciende el cara a cara, el espacio, el aquí y el ahora y, sobre todo, tipifica experiencias. El lenguaje genera campos semánticos y posibilita la acumulación de conocimientos.

La adquisición del conocimiento se mueve entre el ámbito de la experiencia inmediata y la sedimentación, en lo que se llama conocimiento acumulado (stock of knowledge). Un fundamento importante para la comprensión social del mundo de la vida (life-world) es que siempre se presupone que el otro tiene una inteligibilidad y experiencia similar a la mía (Schutz \& Luckmann, 1973). Eso significa que mi comprensión se basa en una integración (o reducción) del otro en lo que se llaman unidades de sentido, que forman mi conocimiento. Esta unión (y reducción) de sentido, que llamamos contextos de sentido (meaning-contexts), es una síntesis de las experiencias directas e inmediatas con otros/objetos y mi explicación (mediada) derivada de mi conocimiento acumulado sobre el mundo social y natural.

El conocimiento se da por medio de marcos semánticos que establecen, respecto a las experiencias, lo que es familiar y lo que es distante. En relación a la construcción social de la naturaleza, la familiaridad con esa realidad estará dada por el tipo de relaciones sociales que se tengan con los ecosistemas. Lo que para un campesino es muy familiar, por ejemplo, el conocimiento de hierbas medicinales, para el funcionario del ministerio de agricultura se trata de algo distante, quizás mediado por sus conocimientos científico-técnicos de las plantas. De esta manera el ambiente no es una naturaleza dada, sino que una construcción social que en cada caso debe ser decodificada para su comprensión. El conocimiento ambiental 
es pues una forma de construcción social de un conocimiento experiencial o sistemático acerca de la naturaleza en que los hombres viven y la relación hombres-naturaleza, en las coordenadas espacio-temporales que son dadas.

Considerando lo anterior, se puede comprender que existen formas de conocimiento acerca de la naturaleza y la ecología (Dryzek, 2005; Hannigan, 2006) que van desde las ecologías antropocéntricas modernizadoras, hasta las ecologías cosmológico-simbólicas, pasando por las ecologías biocéntricas, y sociobiocéntricas (Estenssoro, 2016). Pero como hemos podido verificar en otras investigaciones sobre controversias socioambientales en torno a proyectos en la megaminería, como el caso de la triada agua-energía-minería (Parker, Baigorrotegui, \& Estenssoro, 2016), no sólo se trata de modelos de representación de las formas en que los recursos naturales (agua-energía) son producidos/consumidos de manera sustentable o insustentable, sino que, más allá de ello, en la construcción de los modelos en que subyacen cosmovisiones sobre la ecología y el medio ambiente, pero que además tienen estructuras discursivas y lingüísticas diferenciadoras. Llamaremos desafío del lenguaje al hecho de que más allá de categorías distintas, existen formas de construcción de conocimientos-mediados lingüísticamente- cuya divergencia se torna aguda. Es esta una de nuestras presunciones que están a la base de nuestra tesis, y es que, en los procesos de evaluación ambiental, donde confluyen prácticas institucionales y regulatorias, prácticas socio-productivas, conocimientos tecno-científicos especializados, prácticas y conocimientos cotidianos de comunidades y grupos de base involucrados, hay formas distintas de construcción de conocimientos que son sustancial y semióticamente diferentes.

En este escenario, se propone un marco analítico que incluye diversas formas generales de conocimientos y cuyos contenidos sustantivos han sido clasificados bajo criterios heterodoxos. De acuerdo con este enfoque, (a) en primer lugar, referiremos al conocimiento ambiental, que considera todos aquellos predicados acerca de la naturaleza y de la relación de esta con el hombre. La adquisición de este tipo de conocimiento se mueve entre la experiencia inmediata y la acumulación de esta. (b) En segundo lugar, tenemos el conocimiento sociotécnico, que corresponde a aquel conocimiento especializado, monopolio de expertos. Esta construcción nos interesa, por cuanto se ha dicho que los conocimientos ambientales requieren conocimiento experto, debido a que los problemas ambientales son analizados desde una perspectiva científica. Los expertos científicos poseen injerencia directa y privilegiada en la definición y evaluación de los problemas ambientales, aun cuando los actores sociales no expertos también emplean en forma explícita o tácita este tipo de conocimientos.

En cualquier caso, el conocimiento es un factor interviniente de manera fundamental en los procesos de evaluación ambiental, en todo el ciclo de los proyectos, desde su planificación hasta su implementación. Ahora bien, los tipos de conocimiento sociotécnico, en un plano específico $\mathrm{y}$ en un plano operacional, que intervienen 
pueden distinguirse como cuatro: físicoambiental y de los ecosistemas; técnico-experto (costo-beneficio, alternativas tecnológicas y normas y protocolos de intervención); sociopolítico (por cuanto los proyectos redistribuyen poder y recursos); y culturales (los megaproyectos afectan modos de vida, tradiciones e identidades locales).

Los dos tipos primero mencionados están cargados hacia el polo técnico, aun cuando su connotación social no puede ser negada. Los otros dos tipos, el sociopolítico y el sociocultural, se ubican en un estatus epistémico diferente: si bien en el sentido común se trata de conocimientos que inciden en la vida cotidiana y en la esfera pública (sin una connotación de especialización), en los procesos de evaluación y en los conflictos las formas subyacentes de conocimientos sociopolíticos y aquellas subyacentes de conocimientos socioculturales, pueden llegar a ser decisivas el momento de orientar un curso de acción colectiva o de encontrar soluciones alternativas. Ambas formas de conocimientos se manifiestan en diversos planos desde los más implícitos y espontáneos (predominante en el sentido común local) hasta los más sistemáticos e institucionalizados en los grupos de consultores y asesores (expertos orgánicos de las grandes empresas y de las esferas de poder político).

En cuanto al conocimiento sociotécnico en las evaluaciones ambientales la investigación empírica nos ha mostrado cuán importante resultan los discursos jurídico-normativos. En efecto, de acuerdo a la teoría de De Souza Santos (2003), el derecho y el lenguaje jurídico estarían completamente sujetos o colonizados por la racionalidad cognitivo-instrumental. Coincidentemente, los estudios acerca de las prácticas sociales en torno a la elaboración e implementación de las políticas públicas (Silva, 2009) incluyendo las políticas medioambientales, muestran que estas estarían dominadas por la tecnocracia, esto es, por la hegemonía de un conocimiento experto donde las elites ingenieriles, economicistas y de las ciencias administrativas llevan el pandero.

Complejidad, asimetrías y diversidad de actores en la construcción de conocimiento ambiental

Como se desprende de las reflexiones previas, los tipos y formas de conocimiento que intervienen en un proceso de evaluación y en los conflictos ambientales son múltiples y la dinámica de la interacción social en la que ellos intervienen no es sencilla, por lo que el análisis debe saber distinguir con claridad acerca de cuáles son los múltiples conocimientos de los cuales estamos hablando, pero sobre todo, no solo del plano del contenido, sino que también del plano de la lógica de su construcción semiótica.

Debemos distinguir los conocimientos sistemáticos derivados de la producción institucionalizada de la ciencia y la tecnología, de los conocimientos prácticos que estructuran la vida diaria. Existe de plano una distancia distinguible entre el conocimiento experiencial y el conocimiento sistemático cuya diferencia nos posibilita aquí introducir nuestra reflexión acerca de una serie de distinciones que nos parecen claves para la comprensión de los 
lenguajes-conocimientos en la construcción social del medio ambiente.

En primer lugar, tenemos el conocimiento tácito desde la teoría de Polanyi (1966), que plantea una distinción respecto al conocimiento explícito. Se trata de reconocer formas de conocimiento no dichas ni intencionales, que por lo demás operan en procesos prácticos y evaluativos de la vida. Para este autor, el pensamiento no solo es necesariamente intencional, sino que también está necesariamente lleno de "las raíces que encarna" (p. 7). Uno siempre sabe más de lo que puede decir y no siempre lo que se sabe se puede poner en palabras. Como el conocimiento tácito suele ser no verbal, solo se accede a él por medio de las connotaciones del lenguaje verbal y revela situaciones existenciales mucho más que circunstancias institucionales. ¿Cuán decisivo puede ser y cuánto pesan las formas de conocimiento tácito en los procesos de evaluación ambiental y en sus resoluciones, en las diversas instancias, funcionarios-administradores, promotores, consultores, comunidad local?

En segundo lugar, se plantea lo que Berger y Luckmann (1966|1999) llaman áreas de opacidad del lenguaje y del conocimiento cotidiano. También Latour (2012) nos habla de la opacidad del pensamiento moderno occidental cuando se autoproclama como el pensamiento del progreso y descalifica por irracionales a los conocimientos tradicionales, pero no se autocuestiona las propias irracionalidades que le habitan. ¿Acaso no existen numerosas instancias en la producción del conocimiento sociotécnico en torno a la evaluación ambiental en los cuales existe una no reconocida opacidad, es decir, puntos ciegos del conocimiento que deben ser encubiertos o bien pasados por alto por la lógica burocrática o experta?

Por último, tenemos el conocimiento negativo y la aproximación liminal. En el proceso de producción del conocimiento científico de vanguardia y de alto nivel existen - de acuerdo a los estudios de Knorr-Cetina (2003)- fenómenos liminales, "cosas que no son objetos empíricos de conocimiento positivo ni tampoco regiones informes de lo desconocido, sino algo entre ambos" (Knorr-Cetina, 2003, p. 63). Se trata de áreas en torno al error y la incertidumbre o bien en torno a la corrección de los datos que se necesitan para formar otras maneras de hacer significativos los espacios de error o de incertidumbre en el conocimiento científico. ¿Cuánto de esos procedimientos no están en práctica en los procesos de evaluación ambiental que buscan afirmar su tecnicidad a toda prueba por más que subsisten un conjunto de situaciones liminales?

$\mathrm{Al}$ reconocer la condición liminal de los fenómenos, podemos observar la construcción de conocimiento ambiental como proceso situado y controversial. De allí nuestro interés en analizar los contextos en los cuales se desenvuelven los conflictos ambientales. Se trata, antes que nada, de conflictos entre actores sociales en escenarios específicos, en este caso escenarios socioambientales donde se evidencian asimetrías en las posiciones sociales y de poder de los actores en juego. Por ello nuestra propuesta significa alejarse de la manera clásica en que la ciencia social ha enfocado la problemática del conocimiento en estos casos, como simple 
conocimiento sociotécnico involucrado en los procesos de innovación tecnológica.

Podemos distinguir dos grandes tipos de actores sociales involucrados en la evaluación ambiental de proyectos. Se trata de actores que puede ser mirados con un enfoque desde arriba, es decir, aquellos que intervienen desde el poder o que bien pueden ser analizados desde abajo, es decir, actores que manifiestan su iniciativa desde la propia comunidad local, es decir, en posiciones de poder subordinadas. En este sentido se distinguen dos grupos, primero los actores locales, que son aquellos que están inmersos -por su voluntad propia o no- en proyectos que involucran el medioambiente ya que habitan esos ecosistemas y por lo general se ubican en instancias de poder subordinado. En segundo lugar, tenemos a los denominados actores estratégicos, sujetos cuya su influencia es global y desde arriba, tienen relación directa en la toma de decisiones dentro de conflictos socioambientales y estas afectan a las localidades, modificando las condiciones ambientales y de habitabilidad de su espacio (Parker, 2018).

En los conflictos ambientales se expresa con regularidad una asimetría de posiciones sociales de los actores, donde unos cuantos se ubican en posiciones de poder dominante -generalmente los actores estratégicos- $\mathrm{y}$ otros -generalmente representativos de una densidad demográfica mayor- se ubican en posiciones de poder subordinadas o derechamente en condiciones de dominación -como generalmente ocurre con las comunidades y actores locales.
Esta asimetría resulta ser decisiva en la forma en cómo se van desarrollando los conflictos y en su resolución. En dicha desigualdad, el factor conocimiento es clave como diferenciador de posiciones hegemónicas y/o subordinadas. El acceso al conocimiento forma parte de la propia construcción social de la asimetría, que por lo demás representa un obstáculo para la gobernanza y la resolución democrática de los conflictos. El adecuado diagnóstico de las asimetrías de conocimientos y una propuesta de arreglos consensuados en las negociaciones debidamente conducidas no solo tendería a la reducción de esas asimetrías sino a la generación de condiciones de arreglos viables y que garanticen los derechos humanos, sociales, culturales y ambientales de las poblaciones afectadas.

Para avanzar en esta dirección, sin embargo, es preciso tensionar lo que tradicionalmente se plantea como condición de legitimidad del conocimiento ambiental: la experticia técnica y profesional. En efecto, la relación entre tecnocracia y medio ambiente ha sido vinculada al carácter científico de los problemas ambientales. Por ese carácter se tiende al privilegio de los expertos científicos en la definición y evaluación de los problemas ambientales (Wesselink, Buchanan, Georgiadou, \& Turnhout, 2013). Se ha estudiado en diversos contextos el rol de los expertos y del conocimiento tecnológico en conflictos ambientales (Pellizzoni, 2011) y en la interface ciencia-política en la gobernanza ambiental (Wesselink et al., 2013) mostrándose cómo el conocimiento científico puede llegar a perder legitimidad en las 
tensiones generadas en los procesos de evaluación ambiental (Barandiaran, 2015). Pero poco se ha estudiado las mediaciones sociolingüísticas y semióticas de construcción del conocimiento sociotécnico en esos contextos.

En la literatura hay quienes aseguran que las elites tecnocráticas no plantean un problema para la democracia (Burnham, 1957). Las posturas críticas ven, en cambio, en dichas elites un peligro dada su racionalidad instrumental y técnica tendencialmente antidemocrática (Centeno, 1993; Feenberg, 1993).

En el análisis de las elites tecnocráticas en períodos recientes de Latinoamérica (Joignant \& Guell, 2011) el concepto tecnopols se ha propuesto como una herramienta para comprender nuevas formas de la relación entre conocimientos técnicos y política en los procesos democráticos. Al respecto la distinción que hace Joignant (2011) es relevante. Aunque no ha sido aplicada a los procesos de evaluación ambiental, esta categoría nos parece pertinente para ser aplicada a ellos. Con todo, es necesario complejizar el análisis cuando la división del conocimiento no parece ser tajante entre expertos-técnicos y organizaciones no expertas (analfabetas científicamente) ya que, como ha mostrado Tironi (2012), en la interacción se dan procesos de intercambio y tensiones y se renegocian y redefinen contornos epistémicos.

El análisis científico social debe alejarse de los discursos unidimensionales y monolíticos que sacralizan la verdad científica y relegan los otros conocimientos a un estatus secundario, irrelevante o sencillamente lo descalifican por irracional. Por una restricción mental, un poco perversa, se acuerda de un lado la posibilidad de conocimientos diversos, pero de otro se retiran todas las legitimaciones de verdad a discursos otros que acceden a la realidad por otras vías de representaciones (Latour, 2012).

No es la verdad de la ciencia y la tecnología, es la verificación de la realidad por las instituciones que construyen predicados de realidad capaces de obtener, por medio de complejos procesos y métodos, consensos intersubjetivos. La construcción social del conocimiento ambiental supone una diversidad de instituciones y es esta diversidad la que debe ser revelada y mostrada a fin de que los discursos evaluativos sobre el impacto ambiental adquieran el estatuto cognoscitivo que los aleje del tecnocratismo y los acerque a las posibilidades de la participación ciudadana.

$\mathrm{Al}$ contrario de lo que se presenta como un dato de realidad, a saber, que el conocimiento sociotécnico sería monopolio de los especialistas y expertos, resulta interesante plantarse la cuestión de otra manera, es decir, mirar la producción social de conocimientos en términos de campo (Bourdieu, 1975) de intereses y de lucha por el poder y por la autoridad científica. De lo que se trata es de abordar desde una perspectiva epistemológica y sociológica que busca salir de la trampa del enfoque clásico que hasta el momento se contenta con erigir a un tipo de conocimiento -el racional y científico-técnicocomo el único con valor de verdad.

El análisis del uso de conocimientos sociotécnicos en contextos de evaluación ambiental debe considerar el hábitat institucional en el cual se desarrollan los procesos de evaluación y el 
contexto general de la política pública. Como hemos mostrado en otros trabajos, la política pública esta generalmente diseñada sobre la base de la especialización del conocimiento (Parker, 2014) y por ello sigue la lógica de la sectorialización, siendo incapaz de una perspectiva holística como lo requiere una evaluación de la sustentabilidad ambiental. La división del trabajo genera condiciones para la separación de los conocimientos y la distinción entre científico-técnicos y no expertos (Yearley, 2000) excluyendo cualquier otro tipo. Dicho de otra manera, en todo proceso de evaluación ambiental existen siempre representaciones sociotécnicas -con su lenguaje racionalinstrumental- e intermediaciones burocráticas insalvables -con sus lenguaje racionalregulatorios- y estas últimas tienden a predominar (Latta, 2007; Lostarnau et al., 2011). Las asimetrías en el manejo del conocimiento sociotécnico y en el empleo de formas locales de conocimiento derivan de una asimetría de poder. Las grandes empresas transnacionales o nacionales que son las impulsoras del megaproyecto, así como las entidades que representan el aparato del estado en cada negociación (ministerios, servicio de evaluación ambiental, secretarías regionales ministeriales, municipios, parlamentarios) cuentan con las capacidades y los recursos para imponer su discurso y sus decisiones. Las comunidades locales generalmente desprovistas de acceso a formas científico-técnicas de conocimientos y sobre todo desconociendo el aparataje jurídico-normativo, se muestran vulnerables y subordinados a la imposición de criterios y decisiones que normalmente adquieren un sesgo tecnocrático: precisamente para legitimar, en función de la técnica, una decisión que tiene siempre una connotación política y que afecta la redistribución del poder y de los recursos a nivel de la localidad intervenida por el proyecto.

En todo proceso de negociación de conocimientos que involucra la evaluación ambiental se da una hegemonía del conocimiento que viene desde arriba, esto es de aquel conocimiento que se presenta como técnico o incluso que se presenta como político, es decir que proviene de argumentaciones de tipo económico (fundamentando la inversión), tecnológico (fundamentando la opción tecnológica), normativo (basándose en una interpretación de las leyes existentes) o político (fundamentando en la política del gobierno de turno) y que se ven privilegiadas respecto al conocimiento que proviene desde abajo, es decir, del conocimiento y cultura local que busca asegurar las mejores condiciones de supervivencia y de calidad de vida para los habitantes de la comunidad en cuestión. Finalmente, es preciso tener en cuenta que los contextos de evaluación ambiental se dan muchas veces en el marco de relaciones interculturales. El mapa de conflictos ambientales de Chile y América Latina revela que gran parte de los casos en que inversiones (minería, energía, infraestructura, etc.) de alta complejidad y magnitud se hacen en áreas geográficas con alta densidad de población indígena. En Chile al menos un tercio de los conflictos socioambientales latentes se dan en contexto de territorios indígenas (INDH, 2019). 
La propuesta de Latour (2012) plantea que más allá de las racionalidades debemos estudiar formas de existencia, es coherente con el análisis de que la ciencia-técnica ha sido un conocimiento dominante en la expansión del modo dominante occidental. La revisión de las formas de conocimiento a la luz de la interculturalidad presente en nuestras sociedades y en especial las sociedades periféricas, se hace necesaria. De hecho, existen formas de conocimientos no occidentales que deben ser también reconocidas como, por ejemplo, las formas de valorización simbólica de la naturaleza que tienen los discursos indígenas frente a la mercantilización que hace el discurso y la política extractivista (Parker, 2017).

El conocimiento sociotécnico, en tanto tal, no está distribuido equitativamente y se erige como saber absoluto y verdadero cuestionando otros saberes. Una alternativa es concebir al conocimiento científico-técnico en un amplio espectro de conocimientos sociotécnicos donde caben explicaciones alterativas e incluso sabidurías ancestrales como formas de relacionarse y transformar el mundo y los sistemas ecológicos, lo que De Souza Santos (2010) llama ecología de saberes. Un enfoque intercultural en los diálogos necesarios para hacer avanzar la evaluación ambiental, especialmente en contextos indígenas o de minorías étnicas, es fundamental para que esta evaluación sea conducente y se evite una escalada de conflictos que muchas veces se reducen a fallas de comunicación e incomprensiones y descalificaciones entre actores en posiciones asimétricas.
Elementos de un marco metodológico: análisis crítico y hermenéutico de los discursos ambientales

Los estudios de conflictos socioambientales son normalmente abordados desde la perspectiva de estudios de caso, enfocándose en los fenómenos en donde se presentan movilizaciones de oposición a los proyectos, en especial cuando estos son de gran envergadura. Si bien existe una importante producción académica respecto a conflictos medioambientales en Chile luego del retorno a la democracia, es un ejercicio más provechoso la posibilidad de sumar más casos y analizarlos en conjunto (Maillet \& Albala, 2018). Considerando lo anterior, ha existido una proliferación de estudios que implican analizar los discursos de los actores desde una perspectiva semiótica, a fin de encontrar nuevas maneras de abordar los conflictos socioambientales (Frich \& Espejel, 2006).

Toda estructura semántica del discurso (Barthes, 1966; Greimas, 1966, 1970; Ricoeur, 1986; Van Dijk, 2008) opera sobre la base del encadenamiento progresivo de significantes/significados que van desde los fonemas y semantemas hasta los lexemas y sintagmas, luego estructuras lexemáticas y paradigmáticas y finalmente las estructuras semióticas cosmovisionales. A nivel de las estructuras manifiestas encontramos que los significantes del discurso son las estructuras sintagmáticas de una parte, cuyos significados pueden ser comprendidas -en un enfoque sociolingüístico- como los códigos restringidos y elaborados de que nos habla Berstein (2003). En 
cuanto a las estructuras paradigmáticas del discurso -aquellas que conforma su estructura latente- un enfoque lingüístico clásico no distinguiría un desdoblamiento, que para nuestra concepción teórica actual es fundamental: las formas de elaboración de conocimientos de aquellas que podríamos llamar contenidos o categorías sustantivas (esto es, estructuras de significación cosmovisionales) como las que analizan, por ejemplo, Dryzek (2005) y Hannigan (2006). El aporte de Polanyi (1966) acerca del conocimiento tácito, y las consideraciones de Knorr-Cetina (2003) acerca del enfoque liminal, que hemos mencionado más arriba, nos posibilitarán comprender mejor aquello que aquí estamos llamando formas de elaboración de las categorías paradigmáticas en un enfoque que ciertamente abandona el intratexto y necesariamente recurre a interpretaciones extratextuales.
Enfoque semiótico para el análisis del conocimiento socioambiental.

Más allá del contenido sustantivo de los conocimientos sociotécnicos que ya hemos esbozado en los puntos anteriores, la investigación sociológica ambiental que estudie el conocimiento debe abordar las formas sociolingüísticas de tipo semántico que permitan ahondar en los contenidos paradigmáticos y en última instancia en las estructuras semánticas profundas de los discursos representacionales de los actores en circunstancias de evaluación y conflicto.

De esta manera si aplicamos en una matriz una posible clasificación de categorías del conocimiento, pero sobre la base de sus formas estructurales de construcción lingüística y semántica y basándonos principalmente en las categorías que nos proveen Polanyi (1966) y Berstein (2003), tenemos la configuración que se observa en la Tabla 1.

Tabla1. Categorías formales clasificatorias del conocimiento sociotécnico de los actores sociales

\begin{tabular}{|c|c|c|c|c|c|}
\hline & & \multicolumn{4}{|c|}{ Polanyi } \\
\hline & & \multicolumn{2}{|c|}{ Conocimiento intelectual } & \multicolumn{2}{|c|}{ Conocimiento práctico } \\
\hline & & $\begin{array}{l}\text { Conocimiento } \\
\text { tácito }\end{array}$ & $\begin{array}{l}\text { Conocimiento } \\
\text { Explícito }\end{array}$ & $\begin{array}{l}\text { Conocimiento } \\
\text { tácito }\end{array}$ & $\begin{array}{l}\text { Conocimiento } \\
\text { explícito }\end{array}$ \\
\hline \multirow[t]{2}{*}{ Berstein } & $\begin{array}{l}\text { Códigos } \\
\text { restringidos }\end{array}$ & $\begin{array}{l}\text { Lenguaje vida } \\
\text { cotidiana }\end{array}$ & Formularios & $\begin{array}{l}\text { Saber hacer } \\
\text { cotidiano }\end{array}$ & $\begin{array}{l}\text { Conocimiento } \\
\text { práctico } \\
\text { tecnológico }\end{array}$ \\
\hline & $\begin{array}{l}\text { Códigos } \\
\text { elaborados }\end{array}$ & $\begin{array}{l}\text { Prejuicios e } \\
\text { intuiciones } \\
\text { sistemáticas/ } \\
\text { cosmovisiones } \\
\text { subyacentes }\end{array}$ & $\begin{array}{l}\text { Análisis } \\
\text { científico- } \\
\text { técnico, teorías, } \\
\text { saber experto, } \\
\text { lenguaje } \\
\text { elaborado }\end{array}$ & $\begin{array}{l}\text { Sabiduría } \\
\text { práctica }\end{array}$ & $\begin{array}{l}\text { Teorías- } \\
\text { tecnologías }\end{array}$ \\
\hline
\end{tabular}


Gran parte de las conversaciones e intercambios a nivel de lenguaje de la vida cotidiana vinculados a circunstancias cotidianas estarán estructurados sobre la base de conocimientos tácitos, operando principalmente sobre la base de códigos restringidos ya sea de la vida cotidiana o bien del saber hacer cotidiano. Los funcionarios, los obreros, los dirigentes comunitarios abordarán cada circunstancia en este nivel de registros. Pero cuando se busca una explicitación por medio de formularios, instrumentos y protocolos formales de acción, propios de la implementación de un sistema de evaluación y/o del propio megaproyecto en terreno, se pone en práctica un conocimiento tecnológico al cual solo los obreros especializados, ingenieros en el terreno y funcionarios del sistema ambiental tienen acceso.

Es aquí donde ya comienza a manifestarse la asimetría entre actores de la cual hemos hablado anteriormente y se inicia, ya en este ámbito de los códigos restringidos, un proceso que tiene todo el riesgo de caer en lo que hemos analizado en los apartados previos. En cuanto a los códigos elaborados la distancia puede producirse con mayor evidencia. El conocimiento tácito de las comunidades respecto a las cosmovisiones de la relación hombre-naturaleza, estará desarrollándose en un plano de expresión totalmente distinto al conocimiento científico-técnico de los consultores y expertos del proyecto. La sabiduría práctica, tanto de comunidades como de funcionarios que aplican el proyecto, se ubica generalmente en un plano de inferioridad en cuanto a su injerencia en el proceso, respecto al conocimiento de los ingenieros y expertos tecnológicos. Una temática en la cual hemos verificado en terreno que revela estas diversidades de categorías formales del discurso sociotécnico es el referido al ámbito jurídico y normativo. Muchas veces el manejo que tienen los funcionarios de los protocolos, instrumentos, normas, formularios, entre otros, del orden normativo y que tiene incidencia práctica, pero que encierra un conocimiento intelectual plasmado por medio de códigos restringidos, involucra un tipo de lenguaje jurídico-normativo regulatorio al cual sencillamente no tienen acceso los miembros de las comunidades locales y frente al cual se evidencian totalmente en desventaja.

\section{CONCLUSIONES}

Desde el punto de vista de la sociología ambiental resulta relevante avanzar en el estudio de las formas de representación del medio ambiente, que aquí hemos acotado a las formas de conocimientos ambientales esgrimidos en torno a los procesos de evaluación ambiental. Tomar en consideración la complejidad del aporte de conocimientos de diverso tipo involucrados y los escenarios de asimetrías de posición social y conocimientos en que se encuentran los actores involucrados en la evaluación ambiental podría reducir la posibilidad de que los conflictos se manifiesten de manera irreconciliable. 
Hemos diseñado un marco teórico y metodológico para el estudio de las asimetrías en el conocimiento sociotécnico como factor relevante en los conflictos socioambientales. Nuestro enfoque ha privilegiado una aproximación de tipo lingüístico y discursivo identificando diversos conocimientos sustantivos (ambientales, sociotécnicos e interculturales), complejidades (como la opacidad y liminalidad del conocimiento), estructuras semióticas de base $\mathrm{y}$ formales, junto a diversos formatos paradigmático-semióticos (tácitos / explícitos; con códigos elaborados o restringidos). Todo ello resaltando el riesgo de la tecnocratización del conocimiento y el desafío de la interculturalidad. Esta propuesta forma parte del estudio más amplio sobre las relaciones sociedad-ambiente y de su particular tensión al momento de proceder a lo que la política pública en años recientes llama evaluación ambiental en el contexto de la búsqueda de desarrollos sustentables.

Consideramos que esta propuesta puede entregar valiosas pistas a la política pública ambiental en la medida en que pone de relieve la importancia de la participación ciudadana, de sus alcances, limitaciones y obstáculos, uno de los cuales, de acuerdo con las tesis aquí propuestas, podría residir en las asimetrías y las diversas formas de conocimientos involucrados. Todo lo cual plantea la necesidad de repensar los procesos de evaluación y de la participación ciudadana ambiental en términos de conocimientos sociotécnicos, de participación real y de interculturalidad.

Los resultados de las investigaciones que proponemos sentarían las bases de nuevas formas de instalación de procesos de evaluación en contextos de negociaciones de conocimientos y saberes, asegurando la gobernabilidad ambiental en contextos de actores sociales distintos, asimétricos y en condiciones de interculturalidad latente o abierta, donde el proceso de evaluación ambiental debe asumir la complejidad de contextos y situaciones siempre desafiados por el factor conocimiento.

\section{REFERENCIAS}

Acselrad, H., das Neves Bezerra, G., \& Muñoz Gaviria, E. (2010). Inserción económica internacional y "resolución negociada" de conflictos ambientales en América Latina. EURE (Santiago), 36(107), 27-47. doi: 10.4067/S0250-71612010000100002

Latta, P. A. (2007). Citizenship and the politics of nature: The case of Chile's Alto Bío Bío. Citizenship Studies, 11(3), 229-246. doi: 10.1080/17450100701381805
Barandiaran, J. (2015). Chile's environmental assessments: Contested knowledge in an emerging democracy. Science as Culture, 24(3), 251-275. doi: $10.1080 / 09505431.2014 .992332$

Barandiaran, J. (2016). The authority of rules in Chile's contentious environmental politics. Environmental Politics, 25(6), 1013-1033. doi: 10.1080/09644016.2016.1218156 
Parker, C., \& Pérez Valdivia, J. M. (2019). Asimetría en el conocimiento sociotécnico: Marco teórico para estudiar conflictos medioambientales. Revista de Sociología, 34(1), 4-20. doi: 10.5354/0719529X.2019.54257

Barthes, R. (1966). Introduction à l'analyse structurale des récits. Communications, 8(8), 1-27. doi: 10.3406/comm.1966.1113

Berger, P., \& Luckmann, T. (1966|1999). La construcción social de la realidad. Buenos Aires, Argentina: Amorrortu.

Berstein, B. (2003). Class, codes and control. Theoretical studies towards a sociology of language. Londres, Reino Unido: Routledge.

Bourdieu, P. (1975). La spécificité du champ scientifique et las conditions sociales du progrès de la raison. Sociologies et Société, 71), 91-118. doi: 10.7202/001089ar

Burnham, J. (1957). La revolución de los directores. Buenos Aires, Argentina: Editorial Sudamericana.

Centeno, M. Á. (1993). The new leviathan: The dynamics and limits of technocracy. Theory and Society, 22(3), 307-335. doi: 10.1007/BF00993531

Cordero, E., \& Vargas, I. (2016). Evaluación ambiental estratégica y planificación territorial. Análisis ante su regulación legal, reglamentaria y la jurisprudencia administrativa. Revista Chilena de Derecho, 43(3), 1031-1056. doi: 10.4067/S071834372016000300011

Coria, I. D. (2008). El estudio de impacto ambiental: Características y metodologías. Invenio, 11(20), 125-135.

Costa Cordella, E. (2012). El SEIA en crisis? Conflictos ambientales y ciudadanía. Derecho y Humanidades, 20(1), 357-374. doi:10.5354/0719-2517.2014.34829

Cuadra Montoya, X. (2012). Las trayectorias de la movilización contra HidroAysén: El malestar es sobre lo político, la propuesta es democratizadora. Anuari Del Conflicte Social, 2(1), 1155-1176.

De Souza Santos, B. (2003). Crítica de la razón indolente. Contra el desperdicio de la experiencia, Para un nuevo sentido común: La ciencia, el derecho y la política en la transición paradigmática. Bilbao, Francia: Desclée de Brouwer.
De Souza Santos, B. (2010). Descolonizar el saber, reinventar el poder. Montevideo, Uruguay: Trilce.

Delamaza, G., Maillet, A., \& Martínez Neira, C. (2017). Socio-territorial conflicts in Chile: Configuration and politicization (20052014). ERLACS, 104(1), 23-46. doi: 10.18352/erlacs. 10173

Dryzek, J. S. (2005). The politics of the earth. Environmental discourses. New York, NY: Oxford University Press.

Estenssoro, F. (2016). La "apuesta" ideológicopolítica de los ecologistas: El biocentrismo como nueva expresión de radicalidad y su recepción en Latinoamérica. Revista Direitos Emergentes na Sociedade Global, 5(1), 23-41. doi: 10.5902/2316305425378

Feenberg, A. (1993). The technocracy thesis revisited: On the critique of power. Inquiry, 37(1), 85-102. doi: $10.1080 / 00201749408602341$

Frich, B. A., \& Espejel, B. O. (2006). Semiótica ambiental, y gestión comunitaria. Horizontes Antropológicos, 12(25), 257-269. doi: 10.1590/S0104-71832006000100013

García Leyton, L. (2004). Aplicación del análisis multicriterio en la evaluación de impactos ambientales. (Tesis Doctoral, Universidad Politécnica de Catalunya, Barcelona, España). Recuperada de https://www.tesisenred.net/handle/10803/68 30;jsessionid=D9021D65B6B86D185EC90D 3A6F65D444\#page $=1$

Glasson, J., Therivel, R., \& Chadwick, A. (2005). Introduction to environmental impact assessment. Londres, Reino Unido: Routledge.

Greimas, A. J. (1966). Sémantique structurale. Paris, Francia: Larousse.

Greimas, A. J. (1970). Du sens, essais sémiotiques. Paris, Francia: Du Seuil.

Hannigan, J. (2006). Environmental sociology. New York, NY: Routledge.

Instituto Nacional de Derechos Humanos. (2019). Mapa de conflictos socioambientales 
Parker, C., \& Pérez Valdivia, J. M. (2019). Asimetría en el conocimiento sociotécnico: Marco teórico para estudiar conflictos medioambientales. Revista de Sociología, 34(1), 4-20. doi: 10.5354/0719529X.2019.54257

en Chile. Recuperado de https://mapaconflictos.indh.cl/\#/

Joignant, A. (2011). Tecnócratas, technopols y dirigentes de partido: Tipos de agentes y especies de capital en las elites gubernamentales de la concertación (19902010). En A. Joignant \& P. Guell (Eds.), Notables, tecnócratas y mandarines. Elementos de la sociología de las elites en Chile (1990-2010) (pp. 49-76). Santiago, Chile: Ediciones Universidad Diego Portales.

Joignant, A., \& Guell, P. (2011). Notables, tecnócratas y mandarines. Elementos de la sociología de las elites en Chile (1990-2010). Santiago, Chile: Ediciones Universidad Diego Portales.

Joap, R., \& Clark, B. (2014). Ecology and environment. En M. Sasaki, J. Goldstone, E. Zimmermann, \& S. K. Sanderson (Eds.), Concise encyclopedia of comparative sociology (pp. 457-465). Leiden, Holanda: Brill.

King, L., \& McCarthy, D. (2014). Enrivonmental sociology. Lanham, MD: Rowman \& Littlefield Publishers, Inc.

Knorr-Cetina, K. (2003). Epistemic cultures. How the sciences make knowledge. Cambridge, MA: Harvard University Press.

Latour, B. (2012). Enquête sur les modes d'existence. Une anthropologie des modernes. Paris, Francia: La Découverte.

Lostarnau, C., Oyarzún, J., Maturana, H., Soto, G., Señoret, M., Soto, M., ... \& Oyarzún, R. (2011). Stakeholder participation within the public environmental system in Chile: Major gaps between theory and practice. Journal of Environmental Management, 92(10), 24702478. doi: 10.1016/j.jenvman.2011.05.008

Maillet, A., \& Albala, A. (2018). Conflictos socioambientales en los proyectos eléctricos en Chile (2005-2016): Un análisis configuracional. América Latina Hoy, 79(1), 125-149. doi: 10.14201/alh201879125149
Moraga Sariego, P. (2017). La definición de nuevos estándares en materia de participación ciudadana en el sistema de evaluación de impacto ambiental. Revista Derecho del Estado, 38(1), 177-198. doi: 10.18601/01229893.n38.07

Parker, C. (2014). El mundo académico y las políticas públicas frente a la urgencia del desarrollo sustentable en América Latina y el Caribe. POLIS Revista Latinoamericana, 13(39), 2-17. doi: 10.4000/polis.10469

Parker, C. (2017). Acteurs sociaux et éthiques écologiques dans le conflit extractif, l'éthique, indigène mise en évidence: Une perspective sud-américaine. En N. Brunet (Ed.), L'oeuvre de Pierre Dansereau et l'avenir des sciences de l'environnement. Montreal, Canadá: UQAM.

Parker, C. (2018). Transición energética y actores locales: Una aproximación conceptual. En G. Baigorrotegui \& C. Parker (Eds.), ¿Conectar o desconectar? Energía y comunidad para las transiciones energéticas. Santiago, Chile: Instituto de Estudios Avanzados.

Parker, C., Baigorrotegui, G., \& Estenssoro, F. (2016). Water-energy-mining and sustainable consumption: Views of south american strategic actors. En F. de Castro, B. Hogenboom, \& M. Baud (Eds.), Environmental governance in Latin America (pp. 164-185). Basingstoke, Reino Unido: Parlgrave Macmillan.

Pellizzoni, L. (2011). The politics of facts. Local environmental conflicts and expertise. Environmental Politics, 20(6), 765-785. doi: 10.1080/09644016.2011.617164

Polanyi, M. (1966). The tacit dimension. New York, NY: Dubleday and Co.

Ricoeur, P. (1986). Du texte à l'action. Essais d'herméneutique II. Paris, Francia: Le Seuil.

Schutz, A., \& Luckmann, T. (1973). The structures of the life-world. Evanston, IL: Northwestern University Press. 
Parker, C., \& Pérez Valdivia, J. M. (2019). Asimetría en el conocimiento sociotécnico: Marco teórico para estudiar conflictos medioambientales. Revista de Sociología, 34(1), 4-20. doi: 10.5354/0719529X.2019.54257

Silva, E. (2012). Environment and sustainable development. En P. Kingstone \& D. Yashar (Eds.), Routledge handbook of Latin American politics (pp. 181-199). New York, NY: Routledge.

Silva, P. (2009). In the name of reason: Technocrats and politics in Chile. University Park, PA: Penn State University Press.

Tecklin, D., Bauer, C., \& Prieto, M. (2011). Making environmental law for the market: The emergence, character, and implications of Chile's environmental regime. Environmental Politics, 20(6), 879-898. doi: 10.1080/09644016.2011.617172

Tironi, M. (2012). Pastelero a tus pasteles: Experticias, modalidades de tecnificación y controversias urbanas en Santiago de Chile. En T. Ariztía (Ed.), Produciendo lo social: Usos y transformaciones de las ciencias sociales en el Chile actual. Santiago, Chile: Ediciones Universidad Diego Portales.

Van Dijk, T. (2008). El Discurso como estructura y proceso. Barcelona, España: Gedisa.

Wesselink, A., Buchanan, K. S., Georgiadou, Y., \& Turnhout, E. (2013). Technical knowledge, discursive spaces and politics at the sciencepolicy interface. Environmental Science \& Policy, 30(1), 1-9. doi: 10.1016/j.envsci.2012.12.008

Yearley, S. (2000). Making systematic sense of public discontents with expert knowledge: Two analytical approaches and a case study. Public Understanding of Science, $9(2), 105-$ 122. doi: 10.1088/0963-6625/9/2/302

Manuscrito recibido: 10-05-2019 Manuscrito aceptado: 09-07-2019 